\title{
Supplementary Material to Accompany:
}

\section{Catalytic Coupling of Terminal Alkynes with Isonitriles Promoted by Organoactinide Complexes}

\author{
Eyal Barnea, ${ }^{\dagger}$ Tamer Andrea, ${ }^{\dagger}$ Moshe Kapon, ${ }^{\dagger}$ Jean-Claude Berthet, ${ }^{\ddagger}$ Michel \\ Ephritikhine, ${ }^{\ddagger}$ and Moris S. Eisen ${ }^{*, \dagger}$ \\ ${ }^{\dagger}$ Department of Chemistry and Institute of Catalysis Science and Technology, Technion-Israel \\ Institute of Technology, Haifa, 32000, Israel \\ ¥ Service de Chimie Moléculaire, DSM/DRECAM, CNRS URA 331, CEA Saclay, Cedex 91191, \\ France.
}




\section{Materials and methods}

All manipulations were performed on a high vacuum $\left(10^{-5}\right.$ torr) line, or in a nitrogen filled Vacuum Atmospheres glovebox with a medium capacity recirculator (1-2 ppm $\mathrm{O}_{2}$ ). Argon and Nitrogen were purified by passage through a $\mathrm{MnO}$ oxygen-removal column and a Davison $4 \AA$ molecular sieve column. Hydrocarbon solvents benzene- $d_{6}$ and toluene- $d_{8}$ (Cambridge Isotopes) were distilled under nitrogen from $\mathrm{Na} / \mathrm{K}$ alloy. ${ }^{\mathrm{t}} \mathrm{BuNC}$, ${ }^{\mathrm{t}} \mathrm{BuC} \equiv \mathrm{CH}, \mathrm{TMSC} \equiv \mathrm{CH}, \mathrm{PhC} \equiv \mathrm{CH}$ and ${ }^{\mathrm{i}} \mathrm{PrC} \equiv \mathrm{CH}$ (Aldrich) were degassed and stored under nitrogen over molecular sieves. The actinide complexes $\mathrm{Cp}^{*}{ }_{2} \mathrm{AnMe}_{2}(\mathrm{An}=\mathrm{U}, \mathrm{Th})^{1}$ and $\left[\left(\mathrm{Et}_{2} \mathrm{~N}\right)_{3} \mathrm{U}\right]\left[\mathrm{BPh}_{4}\right]^{2}$ were prepared according to published methods.

NMR spectra were recorded on Advance 500 Bruker spectrometer. Chemical shifts for ${ }^{1} \mathrm{H}$ NMR and ${ }^{13} \mathrm{C}$ NMR are referenced to internal solvent resonances and are reported relative to tetramethylsilane. GC/MS experiments were conducted in a GC/MS (Finnigan Magnum) spectrometer. All reactions were performed in J. Young Teflon valve-sealed NMR tubes.

Crystal structure determination: Single crystal immersed in Paratone-N oil was quickly fished with a glass rod and mounted on the KappaCCD diffractometer under a stream of nitrogen at $230 \mathrm{~K}$. Data collection was carried out with monochromatized MoK $\alpha$ radiation using $\omega$ and $\pi$ scans to cover the Ewald sphere. Accurate cell parameters were obtained with complete collections of intensities and these were corrected in the usual way. The structure was solved by direct methods and completed using successive Fourier difference maps. Refinement was performed anisotropically with respect to the nonhydrogen atoms. Hydrogen atoms were placed at calculated positions and refined using the riding model until convergence was reached.

The following programs were used for the various steps:

1. Data collection: Nonius (1997), KappaCCD 'Collect' Program ${ }^{3}$

2. Cell refinement: HKL Scalepack ${ }^{4}$

3. Data reduction: HKL Denzo and Scalepack ${ }^{4}$

4. Structure solution: S.Mackay, C.J.Gilmore, C.Edwards, M. Tremayne, N. Stuart, K.Shankland "maXus": a computer program for the solution and refinement of crystal 
structures from diffraction data University of Glasgow, Scotland, UK, Nonius BV, Delft, The Netherlands and MacScience Co. Ltd.,Yokohama, Japan (1998).

5. Structure refinement: SHELXL-97. .

6. Molecular graphics:

Molecular Structure Corporation (1999).ORTEP. TEXRAY Structure

Analysis Package. MSC, 3200 Research Forest Drive, The Woodlands, TX 77381, USA.

7. Publication material: SHELXL-97. ${ }^{5}$

\section{References:}

1. Manriquez, J. M.; Fagan, P. J.; Marks, T. J. J. Am. Chem. Soc. 1978, 100, 3939; Bruno, J. W.; Smith, G. M.; Marks, T. J.; Fair, C. K.; Schultz, A. J.; Williams, J. M. J. Am. Chem. Soc. 1986, 108, 40.

2. Berthet, J. C.; Boisson, C.; Lance, M.; Vigner J.; Nierlich, M.; Ephritikhine M. J. Chem. Soc. Dalton Trans. 1995, 3019.

3. Nonius, KappaCCD Server Software; Nonius BV: Delft, The Netherlands, 1997.

4. Otwinowski, Z.; Minor, W. Methods Enzymol. 1997, 276, 307.

5. Sheldrick, G. M. SHELXL97, Program for the refinement of Crystal Structures; University of Gottingen, Germany, 1997. 


\section{Coupling of terminal alkynes and isonitriles}

General procedure - in a typical procedure, a J. Young NMR tube was charged in the glove-box with $\left[\left(\mathrm{Et}_{2} \mathrm{~N}\right)_{3} \mathrm{U}\right]\left[\mathrm{BPh}_{4}\right](7 \mathrm{mg}, 0.009 \mathrm{mmol})$ or $\mathrm{Cp}_{2}{ }_{2} \mathrm{UMe}_{2}(7 \mathrm{mg}, 0.013 \mathrm{mmol})$ or $\mathrm{Cp}_{2}{ }_{2} \mathrm{ThMe}_{2}(5 \mathrm{mg}, 0.01 \mathrm{mmol})$. Alkynes, t-butylisocyanide and $0.5 \mathrm{~mL}$ of solvent $\left(\mathrm{C}_{6} \mathrm{D}_{6}\right.$ or $\left.\mathrm{C}_{7} \mathrm{D}_{8}\right)$ were added by vacuum transfer on a high vacuum line. The sealed tube was heated in an oil bath. The rapid darkening of the solution indicated the formation of the products. Monitoring the reaction was done by ${ }^{1} \mathrm{H}-\mathrm{NMR}$. When the reaction went to completion, the products were identified by NMR and GC/MS analysis of the crude reaction mixture. The solvent and the organic volatiles were transferred to a new NMR tube on high vacuum line. The residues were dissolved in toluene; the solids remaining after the addition of the toluene were separated from the solution by centrifugation.

\section{Coupling of ${ }^{\mathrm{t}} \mathrm{BuNC}$ with TMS-C $\equiv \mathrm{CH}$}

Product 4, 2-methyl- $N$-[3-(trimethylsilyl)prop-2-yn-1-ylidene]propan-2-amine, and

product $\mathbf{5}, \quad N, N^{\prime}$-[(4E)-1-(trimethylsilyl)but-1-yne-3,4-diylidene]bis(2-methylpropan-2amine) were prepared according to the general procedure with the three catalysts:

Catalyst 1 - TMS-C $\equiv \mathrm{CH}(0.06 \mathrm{~mL}, 0.42 \mathrm{mmol})$ and ${ }^{\mathrm{t}} \mathrm{Bu}-\mathrm{N} \equiv \mathrm{C}(0.04 \mathrm{~mL}, 0.35 \mathrm{mmol})$ were added and the reaction mixture was heated to $90^{\circ} \mathrm{C}$ for $48 \mathrm{~h}$ to obtain $4(\mathrm{R}=\mathrm{TMS})$ as the major product ( $80 \%$ yield relative to isonitrile).

Catalyst 2 - TMS-C $\equiv \mathrm{CH}(0.05 \mathrm{~mL}, 0.35 \mathrm{mmol})$ and ${ }^{\mathrm{t}} \mathrm{Bu}-\mathrm{N} \equiv \mathrm{C}(0.07 \mathrm{~mL}, 0.61 \mathrm{mmol})$ were added and the reaction mixture was heated to $100^{\circ} \mathrm{C}$ for $24 \mathrm{~h}$ to obtain 4 ( $\mathrm{R}=\mathrm{TMS}$ ) as the major product in addition to 5 (81 and 19\% yield respectively).

Catalyst 3 - TMS-C $\equiv \mathrm{CH}(0.1 \mathrm{~mL}, 0.7 \mathrm{mmol}$ or $0.2 \mathrm{~mL}, 1.4 \mathrm{mmol})$ and ${ }^{\mathrm{t}} \mathrm{Bu}-\mathrm{N} \equiv \mathrm{C}(0.08$ $\mathrm{mL}, 0.7 \mathrm{mmol}$ ) were added and the reaction mixture was heated to $90^{\circ} \mathrm{C}$ for $23 \mathrm{~h}$ to obtain 4 as the major product ( $85-90 \%$ yield relative to isonitrile). 


\section{Characterization data for the TMS adduct of 4:}

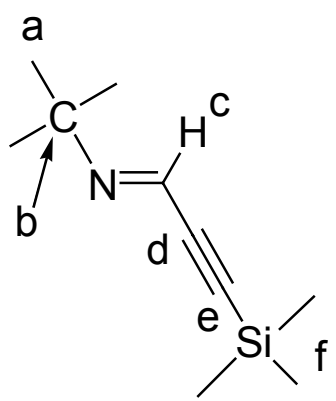

${ }^{1} \mathrm{H}-\mathrm{NMR}\left(500 \mathrm{MHz}, \mathrm{C}_{6} \mathrm{D}_{6}\right): \delta 7.27$ (s, 1H, c), 0.99 (s, 9H, a), 0.10 (s, 9H, f). ${ }^{13} \mathrm{C}-\mathrm{NMR}$ (125 MHz, $\left.\mathrm{C}_{6} \mathrm{D}_{6}\right): \delta 139.6$ (c), 103.8 (d), 96.1 (e), 58.8 (b), 29.2 (a), -0.3 (f). MS (m/z) : $182\left[\mathrm{MH}^{+}\right]$.

\section{Characterization data for TMS adduct of 5:}

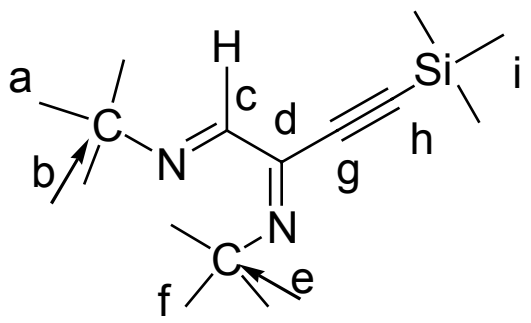

${ }^{1} \mathrm{H}-\mathrm{NMR}\left(500 \mathrm{MHz}, \mathrm{C}_{6} \mathrm{D}_{6}\right): \delta 7.84$ (s, 1H, c), 1.44 (s, 9H, a), 0.96 (s, 9H, f), -0.04 (s, 9H, i).

${ }^{13}$ C-NMR (125 MHz, C $7 \mathrm{D}_{8}$ ): $\delta 158.1$ (c), 149.6 (d), 107.2 (g), 98.3 (h), 57.4 (b), 57.3 (e), 29.1 (a), 28.9 (f), -1.0 (i). 


\section{Coupling of ${ }^{\mathrm{t}} \mathrm{BuNC}$ with ${ }^{\mathrm{i}} \operatorname{Pr}-\mathrm{C} \equiv \underline{\mathrm{CH}}$}

Product 4, 2-methyl- $N$-[4-methylpent-2-yn-1-ylidene]propan-2-amine,

product 5, N,N'-[5-methylhex-3-yne-1,2-diylidene]bis(2-methylpropan-2-amine) and

product 6, $N$-[3-isopropyl-6-methylhept-2-en-4-yn-1-ylidene]-2-methylpropan-2-amine

were prepared according to the general procedure with the three catalysts:

Catalyst $1-{ }^{\mathrm{i}} \mathrm{Pr}-\mathrm{C} \equiv \mathrm{CH}(0.05 \mathrm{~mL}, 0.5 \mathrm{mmol})$ and ${ }^{\mathrm{t}} \mathrm{Bu}-\mathrm{N} \equiv \mathrm{C}(0.04 \mathrm{~mL}, 0.35 \mathrm{mmol})$ were added and the reaction mixture was heated to $90^{\circ} \mathrm{C}$ for $48 \mathrm{~h}$ to obtain $4\left(\mathrm{R}={ }^{\mathrm{i}} \mathrm{Pr}\right)$ as the major product ( $81 \%$ yield relative to isonitrile).

Catalyst $2-1){ }^{\mathrm{i}} \mathrm{Pr}-\mathrm{C} \equiv \mathrm{CH}(0.09 \mathrm{~mL}, 0.9 \mathrm{mmol})$ and ${ }^{\mathrm{t}} \mathrm{Bu}-\mathrm{N} \equiv \mathrm{C}(0.06 \mathrm{~mL}, 0.52 \mathrm{mmol})$ were added and the reaction mixture was heated to $100^{\circ} \mathrm{C}$ for $48 \mathrm{~h}$ to obtain $4\left(\mathrm{R}={ }^{\mathrm{i}} \mathrm{Pr}\right)$ as the major in addition to 5 (75 and 25\% yield respectively). 2 ) ${ }^{\mathrm{i}} \mathrm{Pr}-\mathrm{C} \equiv \mathrm{CH}(0.03 \mathrm{~mL}, 0.3 \mathrm{mmol})$ and ${ }^{\mathrm{t}} \mathrm{Bu}-\mathrm{N} \equiv \mathrm{C}(0.07 \mathrm{~mL}, 0.61 \mathrm{mmol})$ were added and the reaction mixture was heated to $100^{\circ} \mathrm{C}$ for 48 ho to obtain 4 and $5\left(\mathrm{R}={ }^{\mathrm{i}} \mathrm{Pr}\right)$ in equal yields $(100 \%$ overall yield).

Catalyst $3-1){ }^{\mathrm{i}} \mathrm{Pr}-\mathrm{C} \equiv \mathrm{CH}(0.12 \mathrm{~mL}, 1.2 \mathrm{mmol})$ and ${ }^{\mathrm{t}} \mathrm{Bu}-\mathrm{N} \equiv \mathrm{C}(0.13 \mathrm{~mL}, 1.2 \mathrm{mmol})$ were added and the reaction mixture was heated to $90^{\circ} \mathrm{C}$ for $18 \mathrm{~h}$ to obtain 4 as the major product (90\% yield). 2) ${ }^{\mathrm{i}} \mathrm{Pr}-\mathrm{C} \equiv \mathrm{CH}(0.14 \mathrm{~mL}, 1.4 \mathrm{mmol})$ and ${ }^{\mathrm{t}} \mathrm{Bu}-\mathrm{N} \equiv \mathrm{C}(0.08 \mathrm{~mL}, 0.7$ mmol) were added and the reaction mixture was heated to $90^{\circ} \mathrm{C}$. After $17 \mathrm{~h}$, product 4 was obtained (95\% yield) in addition to product 6 (5\% yield). After $210 \mathrm{~h}$, the ratio between 4 and 6 was almost 1:1.

\section{Characterization data for the ${ }^{\mathrm{i}} \mathrm{Pr}$ adduct of 4 :}

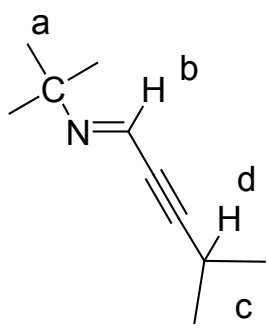

${ }^{1} \mathrm{H}-\mathrm{NMR}\left(500 \mathrm{MHz}, \mathrm{C}_{7} \mathrm{D}_{8}\right): \delta 7.34$ (s, 1H, b), 2.43 (septet, $\left.J=7 \mathrm{~Hz}, 1 \mathrm{H}, \mathrm{d}\right), 1.02$ (s, 9H, a), $1.0(\mathrm{~d}, J=7 \mathrm{~Hz}, 6 \mathrm{H}, \mathrm{c}) .{ }^{13} \mathrm{C}-\mathrm{NMR}\left(75 \mathrm{MHz}, \mathrm{C}_{7} \mathrm{D}_{8}\right)$ [a short relaxation time $(2 \mathrm{sec})$ was used so that the quaternary carbons were not observed]: $\delta 139.7$ (b), 29.2 (a), 22.5 
(c), 21.2 (d). GC-MS: (molecular weight 151) m/z $152\left(\mathrm{M}^{+}+\mathrm{H}\right), 136\left(\mathrm{M}^{+}-\mathrm{CH}_{3}\right), 121\left(\mathrm{M}^{+}-\right.$ $\left.2 \mathrm{CH}_{3}\right), 106\left(\mathrm{M}^{+}-3 \mathrm{CH}_{3}\right), 94\left(\mathrm{M}^{+}-\mathrm{C}_{4} \mathrm{H}_{9}\right), 84\left({ }^{\mathrm{t}} \mathrm{BuNC}^{+}\right)$.

\section{Characterization data for the ${ }^{\mathrm{i}} \mathrm{Pr}$ adduct of 5:}<smiles>[2H]/C(=N/C(C)(C)C)C(C#CC(C)(C)C)=NC([2H])(C)C</smiles>

${ }^{1} \mathrm{H}-\mathrm{NMR}\left(500 \mathrm{MHz}, \mathrm{C}_{7} \mathrm{D}_{8}\right.$ ): $\delta 7.92$ (s, $\left.1 \mathrm{H}, \mathrm{b}\right), 2.45$ (septet, $\left.1 \mathrm{H}, J=7 \mathrm{~Hz}, \mathrm{~d}\right), 1.56$ (s, 9H, a), $1.07(\mathrm{~s}, 9 \mathrm{H}, \mathrm{c}), 1.04(\mathrm{~d}, 6 \mathrm{H}, J=7 \mathrm{~Hz}, \mathrm{e}) .{ }^{13} \mathrm{C}-\mathrm{NMR}\left(125 \mathrm{MHz}, \mathrm{C}_{7} \mathrm{D}_{8}\right)$ [a short relaxation time $(2 \mathrm{sec})$ was used so that the quaternary carbons were not observed]: $\delta 160$ (b), 30.9 (a), 30.8 (c), 23.4 (e), 23.1 (d). MS: (molecular weight 234) m/z $235\left(\mathrm{M}^{+}+\mathrm{H}\right)$, $177\left(\mathrm{M}^{+}{ }^{\mathrm{t}} \mathrm{Bu}\right), 163\left(\mathrm{M}^{+}{ }_{-}^{\mathrm{t}} \mathrm{BuN}\right), 149\left(\mathrm{M}^{+}{ }^{\mathrm{t}} \mathrm{BuNCH}\right), 136\left(\mathrm{M}^{+}-\mathrm{C}_{6} \mathrm{H}_{13} \mathrm{~N}\right), 121\left(\mathrm{M}^{+}-\mathrm{C}_{7} \mathrm{H}_{16} \mathrm{~N}\right)$, $106\left(\mathrm{M}^{+}-\mathrm{C}_{8} \mathrm{H}_{19} \mathrm{~N}\right), 94\left(\mathrm{M}^{+}-\mathrm{C}_{9} \mathrm{H}_{19} \mathrm{~N}\right)$.

\section{Characterization data for the ${ }^{\mathrm{i}} \mathrm{Pr}$ adduct of 6 :}

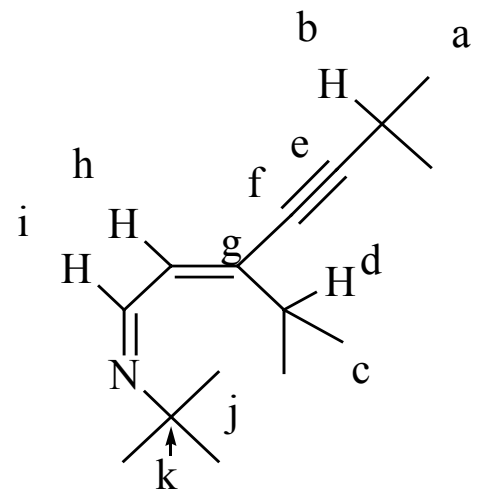

${ }^{1} \mathrm{H}-\mathrm{NMR}\left(500 \mathrm{MHz}, \mathrm{C}_{6} \mathrm{D}_{6}\right): \delta 8.59$ (d, $\left.1 \mathrm{H}, J=8.9 \mathrm{~Hz}, \mathrm{i}\right), 6.53(\mathrm{~d}, 1 \mathrm{H}, J=8.9 \mathrm{~Hz}, \mathrm{~h}), 2.52$ (m, $2 \mathrm{H}, J=7.1$, b and d), 1.20 (s, 9H, j), $1.03\left(\mathrm{~d}, 12 \mathrm{H}, J=7.1 \mathrm{~Hz}\right.$, a and c). ${ }^{13} \mathrm{C}-\mathrm{NMR}$ $\left(125 \mathrm{MHz}, \mathrm{C}_{7} \mathrm{D}_{8}\right)$ [a short relaxation time $(2 \mathrm{sec})$ was used so that the quaternary carbons were not observed]: $\delta 162.9$ (i), 141 (h), 117 (g), 89.3 (f), 87.5 (e), 52.8 (k), 32.5 (j), 26.4 (d), 23.4 (a), 20.4 (a), 20.3 (c). MS: (molecular weight 219) $\mathrm{m} / z 220\left[\mathrm{MH}^{+}\right]$. 


\section{Coupling of ${ }^{\mathrm{t}} \mathrm{BuNC}$ with $\mathrm{PhC} \equiv \underline{\mathrm{CH}}$}

Product 4, 2-methyl- $N$-[3-phenylprop-2-yn-1-ylidene]propan-2-amine

and product 5, $N, N^{\prime}$-[(4E)-1-phenylbut-1-yne-3,4-diylidene]bis(2-methylpropan-2-amine) were prepared according to the general procedure with the three catalysts:

Catalyst 1 - $\mathrm{Ph}-\mathrm{C} \equiv \mathrm{CH}(0.05 \mathrm{~mL}, 0.45 \mathrm{mmol})$ and ${ }^{\mathrm{t}} \mathrm{Bu}-\mathrm{N} \equiv \mathrm{C}(0.05 \mathrm{~mL}, 0.42 \mathrm{mmol})$ were added and the reaction mixture was heated to $90^{\circ} \mathrm{C}$ for $48 \mathrm{~h}$ to obtain $4(\mathrm{R}=\mathrm{Ph})$ as the major product ( $81 \%$ yield relative to isonitrile).

Catalyst $2-\mathrm{Ph}-\mathrm{C} \equiv \mathrm{CH}(0.08 \mathrm{~mL}, 0.78 \mathrm{mmol})$ and ${ }^{\mathrm{t}} \mathrm{Bu}-\mathrm{N} \equiv \mathrm{C}(0.06 \mathrm{~mL}, 0.52 \mathrm{mmol})$ were added and the reaction mixture was heated to $100^{\circ} \mathrm{C}$ for $2 \mathrm{~h}$ to obtain $4(\mathrm{R}=\mathrm{Ph})$ as the major product in addition 5 (90 and 10\% yield respectively).

\section{Characterization data for the $\mathrm{Ph}$ adduct of 4 :}

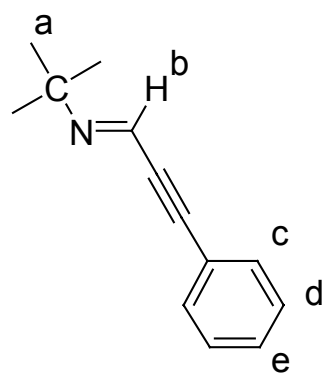

${ }^{1} \mathrm{H}-\mathrm{NMR}\left(300 \mathrm{MHz}, \mathrm{C}_{7} \mathrm{D}_{8}\right): \delta 7.46(\mathrm{~s}, 1 \mathrm{H}, \mathrm{b}), 7.35(\mathrm{~m}, 2 \mathrm{H}, \mathrm{c}), 6.94(\mathrm{~m}, 1 \mathrm{H}, \mathrm{e}), 6.92(\mathrm{~m}$, $2 \mathrm{H}, \mathrm{d}), 1.07$ (s, 9H, tBu).

\section{Characterization data for the $\mathrm{Ph}$ adduct of 5:}

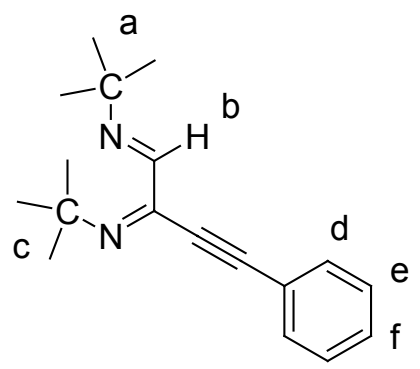

${ }^{1} \mathrm{H}-\mathrm{NMR}\left(300 \mathrm{MHz}, \mathrm{C}_{7} \mathrm{D}_{8}\right): \delta 7.98$ (s, 1H, b), 7.35 (m, 2H, d), 6.94 (m, 1H, f) $6.92(\mathrm{~m}$, 2H, e), 1.58 (s, 9H, a), 1.14 (s, 9H, c). 


\section{Crystal data and structure refinement for 2.}

\begin{tabular}{|c|c|}
\hline Empirical formula & C22 H36 U \\
\hline Formula weight & 538.54 \\
\hline Temperature & $230.0(2) \mathrm{K}$ \\
\hline Wavelength & $0.71073 \mathrm{~A}$ \\
\hline Crystal system, space group & Tetragonal, \\
\hline Unit cell dimensions & 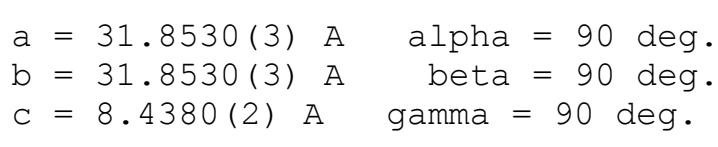 \\
\hline Volume & $8561.3(2) A^{\wedge} 3$ \\
\hline Z, Calculated density & $16, \quad 1.671 \mathrm{Mg} / \mathrm{m}^{\wedge} 3$ \\
\hline Absorption coefficient & $7.583 \mathrm{~mm}^{\wedge}-1$ \\
\hline$F(000)$ & 4160 \\
\hline Crystal size & $0.10 \times 0.12 \times 0.15 \mathrm{~mm}$ \\
\hline Theta range for data collection & 2.50 to $27.46 \mathrm{deg}$. \\
\hline Limiting indices & $-41<=\mathrm{h}<=41, \quad-28<=\mathrm{k}<=28, \quad-10<=1<=9$ \\
\hline Reflections collected / unique & $7964 / 4877$ [R(int) $=0.0282]$ \\
\hline Completeness to theta $=27.46$ & $99.7 \div$ \\
\hline Refinement method & Full-matrix least-squares on $\mathrm{F}^{\wedge} 2$ \\
\hline Data / restraints / parameters & $4877 / 0 / 208$ \\
\hline Goodness-of-fit on $\mathrm{F}^{\wedge} 2$ & 0.909 \\
\hline Final R indices [I>2sigma(I)] & $\mathrm{R} 1=0.0288, \quad w R 2=0.0546$ \\
\hline R indices (all data) & $\mathrm{R} 1=0.0514, \mathrm{wR} 2=0.0575$ \\
\hline Largest diff. peak and hole & 0.439 and -1.390 e. $A^{\wedge}-3$ \\
\hline
\end{tabular}


Table 2. Atomic coordinates $\left(x 0^{\wedge} 4\right)$ and equivalent isotropic displacement parameters $\left(A^{\wedge} 2 \times 10^{\wedge} 3\right)$ for 2 .

$\mathrm{U}(\mathrm{eq})$ is defined as one third of the trace of the orthogonalized Uij tensor.

\begin{tabular}{|c|c|c|c|c|}
\hline & $x$ & y & z & $\mathrm{U}(\mathrm{eq})$ \\
\hline U & $1004(1)$ & $1010(1)$ & $3553(1)$ & $33(1)$ \\
\hline $\mathrm{C}(1)$ & $1252(1)$ & $1816(1)$ & $3036(6)$ & $39(1)$ \\
\hline$C(2)$ & $846(1)$ & $1855(1)$ & $3692(5)$ & $37(1)$ \\
\hline$C(3)$ & $559(1)$ & $1701(1)$ & $2594(6)$ & $40(1)$ \\
\hline$C(4)$ & $780(1)$ & $1572(1)$ & $1249(6)$ & $41(1)$ \\
\hline$C(5)$ & $1214(1)$ & $1644(1)$ & $1521(6)$ & $39(1)$ \\
\hline$C(6)$ & $1656(1)$ & $1986(1)$ & $3754(7)$ & $60(2)$ \\
\hline$C(7)$ & $743(2)$ & 2051 (1) & $5281(6)$ & $58(1)$ \\
\hline$C(8)$ & $86(1)$ & $1728(2)$ & $2710(8)$ & $72(2)$ \\
\hline$C(9)$ & $583(2)$ & $1434(2)$ & $-305(7)$ & $71(2)$ \\
\hline$C(10)$ & $1569(2)$ & $1594(2)$ & $349(7)$ & $67(2)$ \\
\hline$C(11)$ & $1381(1)$ & $229(1)$ & $3870(6)$ & $42(1)$ \\
\hline$C(12)$ & $940(1)$ & $153(1)$ & $3767(6)$ & $43(1)$ \\
\hline$C(13)$ & $810(1)$ & $263(1)$ & $2241(6)$ & $45(1)$ \\
\hline$C(14)$ & $1167(2)$ & $401(1)$ & $1397(6)$ & $51(1)$ \\
\hline$C(15)$ & $1514(2)$ & $386(1)$ & $2423(6)$ & $48(1)$ \\
\hline$C(16)$ & $1660(2)$ & $104(2)$ & $5246(8)$ & $73(2)$ \\
\hline$C(17)$ & $684(2)$ & $-65(2)$ & $5037(7)$ & $68(2)$ \\
\hline$C(18)$ & $365(2)$ & $234(2)$ & $1642(8)$ & $81(2)$ \\
\hline C (19) & $1185(2)$ & $482(2)$ & $-369(7)$ & $91(2)$ \\
\hline$C(20)$ & $1964(2)$ & $505(2)$ & $2033(8)$ & $78(2)$ \\
\hline$C(21)$ & $1479(2)$ & $1115(2)$ & $5732(6)$ & $61(1)$ \\
\hline C (22) & $391(2)$ & $932(2)$ & $5209(7)$ & $68(2)$ \\
\hline
\end{tabular}


Table 3. Bond lengths [A] and angles [deg] for 2.

\begin{tabular}{|c|c|}
\hline $\mathrm{U}-\mathrm{C}(21)$ & $2.404(5)$ \\
\hline $\mathrm{U}-\mathrm{C}(22)$ & $2.415(5)$ \\
\hline $\mathrm{U}-\mathrm{C}(13)$ & $2.694(4)$ \\
\hline $\mathrm{U}-\mathrm{C}(14)$ & $2.709(4)$ \\
\hline $\mathrm{U}-\mathrm{C}(1)$ & $2.723(4)$ \\
\hline $\mathrm{U}-\mathrm{C}(5)$ & $2.733(4)$ \\
\hline $\mathrm{U}-\mathrm{C}(15)$ & $2.738(4)$ \\
\hline $\mathrm{U}-\mathrm{C}(4)$ & $2.739(4)$ \\
\hline $\mathrm{U}-\mathrm{C}(3)$ & $2.742(4)$ \\
\hline $\mathrm{U}-\mathrm{C}(12)$ & $2.742(4)$ \\
\hline $\mathrm{U}-\mathrm{C}(2)$ & $2.743(4)$ \\
\hline $\mathrm{U}-\mathrm{C}(11)$ & $2.774(4)$ \\
\hline$C(1)-C(5)$ & $1.397(6)$ \\
\hline$C(1)-C(2)$ & $1.412(6)$ \\
\hline$C(1)-C(6)$ & $1.521(6)$ \\
\hline$C(2)-C(3)$ & $1.391(6)$ \\
\hline$C(2)-C(7)$ & $1.515(6)$ \\
\hline$C(3)-C(4)$ & $1.398(6)$ \\
\hline$C(3)-C(8)$ & $1.511(6)$ \\
\hline$C(4)-C(5)$ & $1.421(6)$ \\
\hline$C(4)-C(9)$ & $1.519(6)$ \\
\hline$C(5)-C(10)$ & $1.510(6)$ \\
\hline$C(11)-C(15)$ & $1.386(7)$ \\
\hline$C(11)-C(12)$ & $1.426(6)$ \\
\hline$C(11)-C(16)$ & $1.515(7)$ \\
\hline$C(12)-C(13)$ & $1.398(7)$ \\
\hline$C(12)-C(17)$ & $1.516(6)$ \\
\hline$C(13)-C(14)$ & $1.410(6)$ \\
\hline$C(13)-C(18)$ & $1.508(6)$ \\
\hline$C(14)-C(15)$ & $1.405(7)$ \\
\hline C (14) - C (19) & $1.513(7)$ \\
\hline$C(15)-C(20)$ & $1.520(6)$ \\
\hline$C(21)-U-C(22)$ & $94.6(2)$ \\
\hline$C(5)-C(1)-C(2)$ & $108.4(4)$ \\
\hline$C(5)-C(1)-C(6)$ & $125.1(4)$ \\
\hline$C(2)-C(1)-C(6)$ & $126.0(4)$ \\
\hline$C(3)-C(2)-C(1)$ & $108.1(4)$ \\
\hline$C(3)-C(2)-C(7)$ & $126.4(4)$ \\
\hline$C(1)-C(2)-C(7)$ & $125.5(4)$ \\
\hline$C(2)-C(3)-C(4)$ & $108.2(4)$ \\
\hline$C(2)-C(3)-C(8)$ & $126.3(5)$ \\
\hline$C(4)-C(3)-C(8)$ & $124.9(5)$ \\
\hline$C(3)-C(4)-C(5)$ & $108.3(4)$ \\
\hline$C(3)-C(4)-C(9)$ & $125.3(4)$ \\
\hline$C(5)-C(4)-C(9)$ & $126.0(5)$ \\
\hline$C(1)-C(5)-C(4)$ & $107.0(4)$ \\
\hline$C(1)-C(5)-C(10)$ & $125.2(4)$ \\
\hline$C(4)-C(5)-C(10)$ & $127.4(5)$ \\
\hline$C(15)-C(11)-C(12)$ & $107.9(4)$ \\
\hline$C(15)-C(11)-C(16)$ & $126.2(5)$ \\
\hline$C(12)-C(11)-C(16)$ & $125.4(5)$ \\
\hline$C(13)-C(12)-C(11)$ & $107.8(4)$ \\
\hline
\end{tabular}




$\begin{array}{ll}C(13)-C(12)-C(17) & 127.4(4) \\ C(11)-C(12)-C(17) & 124.3(5) \\ C(12)-C(13)-C(14) & 107.7(4) \\ C(12)-C(13)-C(18) & 124.8(5) \\ C(14)-C(13)-C(18) & 127.4(5) \\ C(15)-C(14)-C(13) & 108.1(4) \\ C(15)-C(14)-C(19) & 125.6(5) \\ C(13)-C(14)-C(19) & 125.6(5) \\ C(11)-C(15)-C(14) & 108.4(4) \\ C(11)-C(15)-C(20) & 124.7(5) \\ C(14)-C(15)-C(20) & 126.9(5)\end{array}$

Symmetry transformations used to generate equivalent atoms: 
Table 4. Anisotropic displacement parameters ( $A^{\wedge} 2 \times 10^{\wedge} 3$ ) for 2. The anisotropic displacement factor exponent takes the form:

$-2 \mathrm{pi}^{\wedge} 2$ [ $\mathrm{h}^{\wedge} 2 \mathrm{a}^{\star \wedge} 2 \mathrm{U11}+\ldots+2 \mathrm{~h} \mathrm{k} \mathrm{a}^{\star} \mathrm{b} \mathrm{b}^{\star} \mathrm{U} 12$ ]

\begin{tabular}{|c|c|c|c|c|c|c|}
\hline & U11 & U22 & U33 & U23 & U13 & U12 \\
\hline $\mathrm{U}$ & $40(1)$ & 31 (1) & $29(1)$ & $-1(1)$ & $3(1)$ & $-3(1)$ \\
\hline$C(1)$ & $37(2)$ & $36(2)$ & $44(3)$ & $5(2)$ & $-3(2)$ & $-7(2)$ \\
\hline$C(2)$ & $44(2)$ & $30(2)$ & $36(3)$ & $0(2)$ & $6(2)$ & $2(2)$ \\
\hline$C(3)$ & $34(2)$ & $38(2)$ & $49(3)$ & $3(2)$ & $-2(2)$ & $4(2)$ \\
\hline$C(4)$ & $54(3)$ & $33(2)$ & $37(3)$ & $4(2)$ & $-14(2)$ & $-4(2)$ \\
\hline$C(5)$ & $49(3)$ & $34(2)$ & $35(3)$ & $5(2)$ & $10(2)$ & $-1(2)$ \\
\hline$C(6)$ & $40(3)$ & $55(3)$ & $84(5)$ & $11(3)$ & $-13(3)$ & $-20(2)$ \\
\hline$C(7)$ & $73(3)$ & $47(3)$ & $55(4)$ & $-6(3)$ & $10(3)$ & $-3(2)$ \\
\hline$C(8)$ & $39(3)$ & $82(4)$ & $94(5)$ & $13(4)$ & $-8(3)$ & $-6(3)$ \\
\hline C (9) & $111(5)$ & $51(3)$ & $52(4)$ & $3(3)$ & $-38(3)$ & $-6(3)$ \\
\hline C (10) & $76(4)$ & $61(3)$ & $65(4)$ & $10(3)$ & $34(3)$ & $5(3)$ \\
\hline$C(11)$ & $47(3)$ & $33(2)$ & $45(3)$ & $-5(2)$ & $-9(2)$ & $9(2)$ \\
\hline C (12) & $56(3)$ & $33(2)$ & $41(3)$ & $3(2)$ & $5(2)$ & $2(2)$ \\
\hline C (13) & $57(3)$ & $32(2)$ & $46(3)$ & $-5(2)$ & $-13(2)$ & $2(2)$ \\
\hline C (14) & 87 ( 4) & $34(2)$ & $34(3)$ & $-6(2)$ & $6(3)$ & $6(2)$ \\
\hline$C(15)$ & $53(3)$ & $39(3)$ & $54(4)$ & $-6(2)$ & 12 (3) & $5(2)$ \\
\hline$C(16)$ & $87(4)$ & $66(4)$ & $66(4)$ & $-7(3)$ & $-25(3)$ & $21(3)$ \\
\hline C (17) & $85(4)$ & $48(3)$ & $72(4)$ & $15(3)$ & $19(3)$ & $3(3)$ \\
\hline C (18) & $89(4)$ & $50(3)$ & $102(6)$ & $-17(3)$ & $-50(4)$ & $17(3)$ \\
\hline C (19) & $182(7)$ & $61(4)$ & $30(3)$ & $-4(3)$ & $4(4)$ & $22(4)$ \\
\hline$C(20)$ & $59(3)$ & $62(3)$ & $112(6)$ & $-4(3)$ & $35(4)$ & $4(3)$ \\
\hline$C(21)$ & $84(4)$ & $50(3)$ & $49(4)$ & $3(3)$ & $-17(3)$ & $-8(3)$ \\
\hline$C(22)$ & $76(4)$ & $51(3)$ & $75(5)$ & $0(3)$ & $32(3)$ & $-6(3)$ \\
\hline
\end{tabular}


Table 5. Hydrogen coordinates $\left(x 1^{\wedge} 4\right)$ and isotropic displacement parameters $\left(A^{\wedge} 2 \times 10^{\wedge} 3\right)$ for 2 .

\begin{tabular}{|c|c|c|c|c|}
\hline & $x$ & $\mathrm{y}$ & z & $\mathrm{U}(\mathrm{eq})$ \\
\hline $\mathrm{H}(6 \mathrm{~A})$ & 1600 & 2088 & 4803 & 90 \\
\hline $\mathrm{H}(6 \mathrm{~B})$ & 1761 & 2210 & 3108 & 90 \\
\hline $\mathrm{H}(6 \mathrm{C})$ & 1860 & 1765 & 3803 & 90 \\
\hline $\mathrm{H}(7 \mathrm{~A})$ & 998 & 2133 & 5801 & 88 \\
\hline $\mathrm{H}(7 \mathrm{~B})$ & 596 & 1851 & 5927 & 88 \\
\hline $\mathrm{H}(7 \mathrm{C})$ & 569 & 2294 & 5123 & 88 \\
\hline $\mathrm{H}(8 \mathrm{~A})$ & -38 & 1594 & 1806 & 108 \\
\hline $\mathrm{H}(8 \mathrm{~B})$ & 2 & 2017 & 2739 & 108 \\
\hline $\mathrm{H}(8 \mathrm{C})$ & -6 & 1590 & 3660 & 108 \\
\hline $\mathrm{H}(9 \mathrm{~A})$ & 800 & 1361 & -1044 & 107 \\
\hline $\mathrm{H}(9 \mathrm{~B})$ & 418 & 1660 & -731 & 107 \\
\hline $\mathrm{H}(9 \mathrm{C})$ & 406 & 1195 & -122 & 107 \\
\hline $\mathrm{H}(10 \mathrm{~A})$ & 1465 & 1467 & -604 & 101 \\
\hline $\mathrm{H}(10 \mathrm{~B})$ & 1784 & 1420 & 800 & 101 \\
\hline $\mathrm{H}(10 \mathrm{C})$ & 1685 & 1865 & 105 & 101 \\
\hline $\mathrm{H}(16 \mathrm{~A})$ & 1489 & 9 & 6111 & 109 \\
\hline $\mathrm{H}(16 \mathrm{~B})$ & 1822 & 342 & 5580 & 109 \\
\hline $\mathrm{H}(16 \mathrm{C})$ & 1845 & -117 & 4920 & 109 \\
\hline $\mathrm{H}(17 \mathrm{~A})$ & 852 & -100 & 5972 & 103 \\
\hline $\mathrm{H}(17 \mathrm{~B})$ & 597 & -336 & 4659 & 103 \\
\hline $\mathrm{H}(17 \mathrm{C})$ & 442 & 101 & 5283 & 103 \\
\hline $\mathrm{H}(18 \mathrm{~A})$ & 355 & 325 & 559 & 121 \\
\hline $\mathrm{H}(18 \mathrm{~B})$ & 187 & 409 & 2277 & 121 \\
\hline $\mathrm{H}(18 \mathrm{C})$ & 271 & -52 & 1709 & 121 \\
\hline $\mathrm{H}(19 \mathrm{~A})$ & 907 & 474 & -801 & 137 \\
\hline $\mathrm{H}(19 \mathrm{~B})$ & 1355 & 271 & -867 & 137 \\
\hline $\mathrm{H}(19 \mathrm{C})$ & 1307 & 754 & -557 & 137 \\
\hline $\mathrm{H}(20 \mathrm{~A})$ & 1979 & 604 & 960 & 117 \\
\hline $\mathrm{H}(20 \mathrm{~B})$ & 2142 & 263 & 2152 & 117 \\
\hline $\mathrm{H}(20 \mathrm{C})$ & 2056 & 722 & 2740 & 117 \\
\hline $\mathrm{H}(21 \mathrm{~A})$ & 1758 & 1155 & 5333 & 91 \\
\hline $\mathrm{H}(21 \mathrm{~B})$ & 1473 & 874 & 6412 & 91 \\
\hline $\mathrm{H}(21 \mathrm{C})$ & 1394 & 1359 & 6321 & 91 \\
\hline $\mathrm{H}(22 \mathrm{~A})$ & 149 & 885 & 4555 & 101 \\
\hline $\mathrm{H}(22 \mathrm{~B})$ & 351 & 1182 & 5822 & 101 \\
\hline $\mathrm{H}(22 \mathrm{C})$ & 429 & 697 & 5908 & 101 \\
\hline
\end{tabular}

